\title{
WH, Rat Strain
}

National Cancer Institute

\section{Source}

National Cancer Institute. WH, Rat Strain. NCI Thesaurus. Code C76191.

A Wistar substrain established in Hanover, Germany (1964), this rat breed is a white albino with pink eyes, genotype c. The WH rat has a low incidence of overall spontaneous tumor formation; the most common in males being pituitary adenomas while females exhibit spontaneous mammary, thyroid, and pituitary adenomas. This is a multi-purpose research strain and applications include aging, oncology, toxicology, and safety and efficacy testing. 